\title{
BS Seeker: precise mapping for bisulfite sequencing
}

\author{
Pao-Yang Chen, Shawn J Cokus and Matteo Pellegrini*
}

\begin{abstract}
Background: Bisulfite sequencing using next generation sequencers yields genome-wide measurements of DNA methylation at single nucleotide resolution. Traditional aligners are not designed for mapping bisulfite-treated reads, where the unmethylated Cs are converted to Ts. We have developed BS Seeker, an approach that converts the genome to a three-letter alphabet and uses Bowtie to align bisulfite-treated reads to a reference genome. It uses sequence tags to reduce mapping ambiguity. Post-processing of the alignments removes non-unique and low-quality mappings.
\end{abstract}

Results: We tested our aligner on synthetic data, a bisulfite-converted Arabidopsis library, and human libraries generated from two different experimental protocols. We evaluated the performance of our approach and compared it to other bisulfite aligners. The results demonstrate that among the aligners tested, BS Seeker is more versatile and faster. When mapping to the human genome, BS Seeker generates alignments significantly faster than RMAP and BSMAP. Furthermore, BS Seeker is the only alignment tool that can explicitly account for tags which are generated by certain library construction protocols.

Conclusions: BS Seeker provides fast and accurate mapping of bisulfite-converted reads. It can work with BS reads generated from the two different experimental protocols, and is able to efficiently map reads to large mammalian genomes. The Python program is freely available at http://pellegrini.mcdb.ucla.edu/BS Seeker/BS Seeker.html.

\section{Background}

Epigenetic regulation, such as cytosine (C) DNA methylation, is important in gene regulation and transposon silencing. The gold standard technique for studying DNA methylation is genomic bisulfite sequencing [1]. Sodium bisulfite converts unmethylated Cs to uracils, but 5methylcytosines remain unconverted. Hence, after PCR amplification, unmethylated $\mathrm{Cs}$ are converted to thymines $(\mathrm{T})$ while methylated Cs are unchanged. Recently, Cokus et al. and Lister et al. developed protocols, BS-Seq [2] and MethylC-seq [3], which couple bisulfite sequencing with next generation sequencing and completed a first single nucleotide resolution map of methylation in Arabidopsis. While these approaches open up new avenues for genome-wide measurements of DNA methylation [3-6], aligning millions of bisulfite-treated short reads (BS reads) onto the reference genome remains a challenge. Mapping bisulfite-converted reads leads to

* Correspondence: matteop@mcdb.ucla.edu

1 Department of Molecular, Cell, and Developmental Biology, University of California, Los Angeles, CA, USA

Full list of author information is available at the end of the article ambiguity, since Ts in the read can map to both genomic Cs or Ts. Most alignment tools, such as BLAT [7], SOAP [8], and Bowtie [9] do not explicitly enable bisulfite mapping.

Currently, there are only a few aligners explicitly designed for mapping BS reads. CokusAlignment [2] treats each cycle in a read as probabilities of A, C, T, G and uses a suffix tree searching algorithm. However, to date only the Arabidopsis suffix tree has been published. Other newly-developed bisulfite mapping software includes BSMAP [10], RMAP [11] and MAQ [12], which, unlike CokusAlignment, model reads as discrete base calls instead of probability vectors. BSMAP enumerates all possible combinations of $\mathrm{C} / \mathrm{T}$ conversion in the $\mathrm{BS}$ read to find the uniquely mapping position with the least mismatches on the reference genome. It is reported [10] to have a similar sensitivity as CokusAlignment and outperformed the methods described in [3] and [4]. The bisulfite mapping in RMAP uses Wildcard matching for mapping Ts. MAQ also has a methylation alignment mode and it assigns non-unique reads randomly to one of the best-matching positions. 
Two library protocols have been developed for constructing bisulfite-converted libraries (see Fig 1). Cokus et al's protocol [2] uses two amplification steps: the first amplification generates both forward and reverse bisulfite-converted sequences ligated with DNA adapters of DpnI restriction sites. These sequences are then digested by DpnI restriction enzymes that result in the 5bp sequence tags on the bisulfite-converted sequences. There are two patterns of tags based on the forward $(+\mathrm{FW})$ and reverse (-FW) directions of the bisulfite-converted sequences. After the ligation with standard Solexa adapters and the second amplification step, four types of bisulfite-converted reads are generated. They are forward and reverse reads from Watson $(+F W,+R C)$ and Crick stands (-FW, $-\mathrm{RC}$ ), respectively (see Fig $2 \mathrm{~A}$ ). These tags are essential to reduce the ambiguity of certain classes of reads. Unlike BSMAP, MAQ or RMAP, BS Seeker is able to explicitly use the tags to improve mapping. The second experimental protocol (i.e. Lister et al) generates bisulfite libraries using premethylated adapters, and in this case no tags are present and all reads are $+\mathrm{FW}$ or -FW.

BS Seeker uses Bowtie for mapping BS reads generated from either experimental protocol. It maps $\mathrm{C} / \mathrm{T}$ converted $\mathrm{FW}$ reads to the $\mathrm{C} / \mathrm{T}$ converted reference strands, and G/A converted RC reads to G/A converted, reverse complements of the reference strands. Post-processing removes low-quality mappings based on the number of mismatches. The strategy of bisulfite converting the reference sequence by treating all Cs as Ts (Gs as As) has also been used in $[4,6,13]$, although implementations of this approach have not been published. However, most of these approaches are less precise than the ones we present here as they cannot correctly handle reads that are partially methylated.

For the evaluation of our aligner, we use synthetic BS reads to assess the accuracy of mapping. We evaluate the

\section{Cokus et al's library protocol}

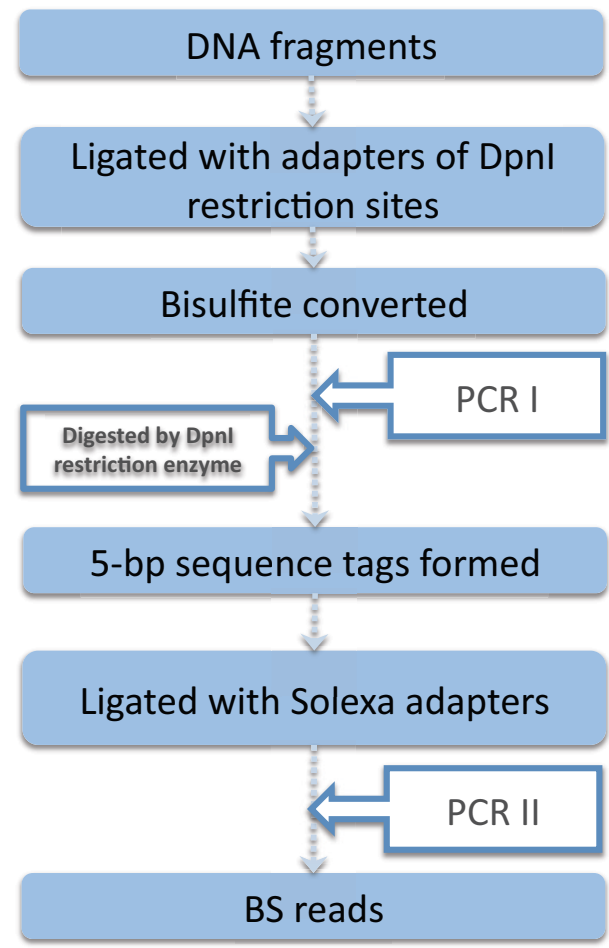

Lister et al's library protocol

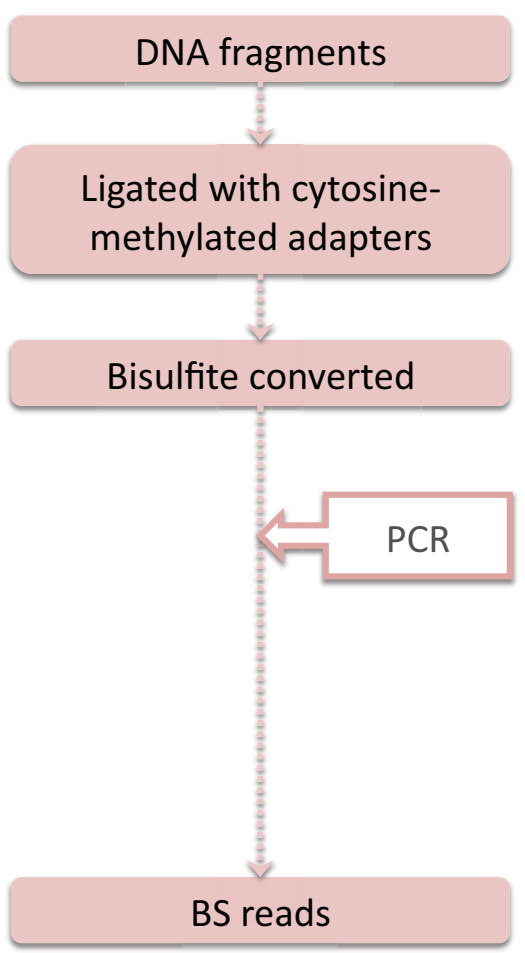

Figure 1 The two library protocols generating bisulfite-converted reads. Cokus et al's experimental protocol uses two amplification steps for generating bisulfite-converted sequences and for high throughput sequencing. The bisulfite-converted reads are preceded by one of two tags in the first 5 nucleotides of reads. Lister et al's protocol generates bisulfite libraries using premethylated adapters, and in this case no tags are present. 
Methylated C Unmethylated C +FW

FW tag

RC tag $\rightarrow$ tctgt AAGTCGTGTTA

$+\mathrm{RC}$

TTCAGCACAAT tacct

Watson (+) $\rightarrow$---- AAGTCGTGCTA

Crick (-) $<----$ TTCAGCACGAT

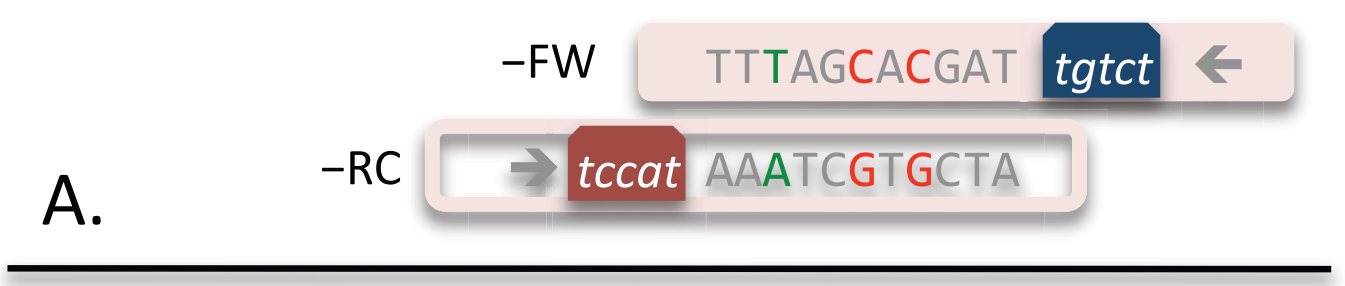

\section{Step 2: Post-processing}

\section{Step 1: 3-letter alignment}

\section{L \\ B.}

BS read: tctgt ACGTCATGTTA

$\mathrm{C} / \mathrm{T} \rightarrow \mathrm{ATGTTATGTTA}$

C/T Watson

$>$---ATGTTGTGTTA

$\rightarrow---A T G T C G T G C T A$

mismatch not a mismatch

mismatch

Figure 2 Schematic diagrams of the 4 forms of BS reads, mapping and post processing. $2 A$. BS reads may be in one of the 4 forms: $+F W,+R C$, FW, -RC. 2B. Bowtie aligns $C / T$ converted reads to the $C / T$ converted strands. During the post processing, the number of mismatches is counted except those between read Ts and genomic Cs. Low-quality mappings with many mismatches are removed. 
mapping results by comparing the methylation statistics in the synthetic reads and in the mapped sequences. We also use BS Seeker to align an Arabidopsis library and human libraries from the two experimental protocols, which provides us with an evaluation using real data.

\section{Implementation}

Depending on the bisulfite experimental protocol that was used to generate the library $[2,3]$, BS reads may be observed in either four or two forms. Cokus et al's protocol generates a forward read $(+\mathrm{FW})$ from the Watson strand, the reverse complement $(+\mathrm{RC})$ of $+\mathrm{FW}$, a forward read (-FW) from the Crick strand, and the reverse complement $(-\mathrm{RC})$ of $-\mathrm{FW}$, see Fig 2A. Lister et al's protocol generates only $+\mathrm{FW}$ and $-\mathrm{FW}$ reads. We first describe how BS Seeker handles data generated from Cokus et al's protocol. It first converts all Cs to Ts on FW reads and both strands of the reference genome, so that the subsequent mapping is performed using only 3 letters, A, T, G. Similarly, G/A conversion is performed on RC reads and both strands of the reverse complement of the reference genome. Then it uses Bowtie to map the $\mathrm{C} / \mathrm{T}$ converted FW reads to the $\mathrm{C} / \mathrm{T}$ converted Watson and Crick strands, and the G/A converted RC reads to the two G/A converted reverse complements of the Watson and Crick strands. Reads that do not have a tag are treated as if they could be both FW and RC reads. During each of the four runs of Bowtie, the mapped positions for each read are recorded. After all the runs of Bowtie are complete, only unique alignments are retained. Here, we define unique alignments as those that have no other hits with - the same or fewer mismatches in the 3-letter alignment (between the converted read and the converted genomic sequence). Finally, we calculate the number of mismatches. For this calculation we consider a read $\mathrm{T}$ that aligns to a genomic $C$ as a match, while a read $C$ that aligns to a genomic $\mathrm{T}$ is considered a mismatch (see Fig 2B). Similarly, when aligning $R C$ reads, a read A that aligns to a genomic $\mathrm{G}$ is considered a match, while a read $\mathrm{G}$ that aligns to a genomic $\mathrm{A}$ is considered a mismatch. Low-quality alignments with the number of mismatches larger than the user-defined value are discarded. Aligning reads generated from Lister et al's protocol is simpler, since there are no $\mathrm{RC}$ reads, and consequently Bowtie is only run twice.

\section{Results and Discussion}

We first tested BS Seeker against three bisulfite aligners, BSMAP, RMAP and MAQ, by mapping synthetic reads. The sensitivity and the specificity of the aligner's output are assessed by calculating the percentage of reads that mapped uniquely and their accuracy, which is the ratio of the number of correctly mapped reads over the total uniquely mapped reads. We also calculate the inferred average methylation rate in order to discern possible mapping bias from the aligners. We then show our mapability on a lane of experimental data from Arabidopsis. Finally, we mapped the BS reads of human libraries generated from the two experimental protocols, in order to measure the performance of BS-Seeker on different protocols, and compare the speed of alignment to RMAP.

\section{Mapping synthetic reads}

We simulated 36-mer BS reads from human chromosome 21. One million contained no base calling errors and another one million had base calling errors that follow the error distribution from [14] (see Supplementary Information in Additional file 1 for details). These were mapped to chromosome 21 using all four aligners. The simulated data were generated using both protocols. As shown in Table 1 and Supplementary Table S1 in Additional file 1, we found that in all cases BSMAP was significantly slower than the other aligners. MAQ's strategy, randomly assigning one of the best-matching positions for non-unique reads, results in a lower accuracy and biased estimates of the methylation rates. When the simulated reads are based on Lister et al's protocol with no tags, RMAP has a slight speed advantage over BS Seeker, but the two methods are otherwise quite similar. However, when the Cokus et al library protocol is used, BS Seeker had a higher accuracy and shorter run time than RMAP, since it is able to explicitly account for the FW/RC tags. Furthermore, the methylation rate inferred from the mapped reads using BS Seeker is very close to that of the initial synthetic data, indicating that the alignments are less biased than those of RMAP. These results suggest that BS Seeker is able to work with data from both protocols. By explicitly using tag information, it is able to optimally align reads generated by the Cokus et al. protocol.

\section{Mapping BS reads from Arabidopsis}

We mapped 2,946,339 BS reads of a single lane of an Arabidopsis library from Cokus, et al., (2008), which is the same library tested in $\mathrm{Xi}$ and $\mathrm{Li},(2009)$. BS Seeker is able to uniquely map $56.3 \%$ of the reads, which compares favorably to the coverage reported in Xi et al. The methylation rates inferred from the mapped reads for CG, CHG and $\mathrm{CHH}$ ( $\mathrm{H}$ stands for $\mathrm{A}$ or $\mathrm{C}$ or $\mathrm{T}$ ) are $25.5 \%, 8 \%$, and $2.2 \%$ respectively, which are very close to the published results [2], i.e, 24\% CG, 6.7\% CHG and 1.7\% CHH methylation.

\section{Mapping BS reads from Human libraries}

In order to test the performance of BS Seeker on large genomes, we used it to map BS reads from human libraries generated from the two protocols [6]. The mapabilities of reads from Cokus et al's protocol and from Lister et al's protocol are very close $(38.3 \%$ and $38.6 \%$ respectively). The CG methylation rate we obtained from the 
Table 1: Mapping 1M synthetic human chr. 21 reads onto human chr. 21

\begin{tabular}{|c|c|c|c|c|c|c|c|}
\hline Aligner & \multirow{2}{*}{$\begin{array}{c}\text { Experimental } \\
\text { protocol }\end{array}$} & \multirow{2}{*}{$\begin{array}{c}\text { Uniquely } \\
\text { (or best a) } \\
\text { mapped } \\
\text { reads b(\%) }\end{array}$} & \multirow{2}{*}{$\begin{array}{c}\text { Accuracy } \\
(\%)\end{array}$} & \multicolumn{3}{|c|}{ Methylation rates c (CG/CHG/CHH) (\%) } & \multirow[t]{2}{*}{ CPU time } \\
\hline \multicolumn{4}{|c|}{ No base calling error } & & & & \\
\hline BS Seeker & Lister et al & 91.7 & 100 & 72.0 & 0 & 0 & $209 s^{d}$ \\
\hline BSMAP & Lister et al & 92.1 & 100 & 72.3 & 0 & 0 & $15 \mathrm{~h} 43 \mathrm{~m} 20 \mathrm{~s}$ \\
\hline RMAP & Lister et al & 91.7 & 100 & 72.0 & 0 & 0 & $185 \mathrm{~s}$ \\
\hline MAQ & Lister et al & $>99.9$ & 93.4 & 67.7 & 0 & 0 & $353 \mathrm{~s}$ \\
\hline BS Seeker & Cokus et al & 89.6 & 100 & 72.0 & 0 & 0 & $263 s^{d}$ \\
\hline BSMAP & Cokus et al & 89.8 & 99.6 & 72.4 & 0 & 0 & $15 \mathrm{~h} 46 \mathrm{~m} 40 \mathrm{~s}$ \\
\hline RMAP & Cokus et al & 80.2 & 99.0 & 71.3 & 0 & 0.1 & $400 \mathrm{~s}$ \\
\hline MAQ & Cokus et al & 73.0 & 92.2 & 68.2 & 0 & 0 & $665 \mathrm{~s}$ \\
\hline \multicolumn{8}{|c|}{ Simulated base calling errors } \\
\hline BS Seeker & Lister et al & 91.2 & 99.54 & 71.5 & 0.4 & 0.4 & $217 s$ \\
\hline BSMAP & Lister et al & 91.1 & 99.57 & 72.1 & 0.4 & 0.4 & $15 \mathrm{~h} 19 \mathrm{~m} 51 \mathrm{~s}$ \\
\hline RMAP & Lister et al & 91.0 & 99.52 & 71.6 & 0.4 & 0.4 & $188 \mathrm{~s}$ \\
\hline MAQ & Lister et al & 99.5 & 92.9 & 67.8 & 0.4 & 0.4 & $340 s$ \\
\hline
\end{tabular}

a BS Seeker, BSMAP, and RMAP discard non-uniquely mapped reads. MAQ keeps non-uniquely mapped reads and assigns them to one of the best-matching positions. b Up to 2 mismatches are allowed. c The simulated methylation rates are set to be $72 \%, 0 \%$, and $0 \%$ for CG, CHG, and

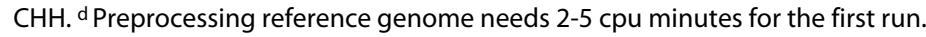

mapping of reads from Lister et al's protocol is $82.3 \%$, which coincides with the published result of $82.7 \%$ [6] (see Supplementary Information in Additional file 1 for the distributions of methylation levels).

We also used RMAP to align the same human libraries. The running time for RMAP to map one lane of reads is between 11.9-13.7 hours, while for BS Seeker it is between 20-50 minutes. Both aligners showed close mapability, see Table 2 . The significant advantage on the mapping efficiency suggests that BS Seeker is the most efficient bisulfite aligner for mapping large genomes.

\section{Conclusions}

We present a tool for mapping BS reads, the BS Seeker. It is simple to operate, and achieves high accuracy and coverage. We have made this tool publicly available for the community.

Table 2: Comparison of mapping efficiency on mapping three lanes of human data

\begin{tabular}{|c|c|c|c|c|c|}
\hline \multirow[t]{2}{*}{ Reads file [6] } & \multirow[t]{2}{*}{ Number of reads } & \multicolumn{2}{|c|}{ Uniquely mapped reads (\%) } & \multicolumn{2}{|c|}{ Running time } \\
\hline & & BS Seeker & RMAP & BS Seeker & RMAP \\
\hline SRR019048 & $15,311,970$ & 40.8 & 41.0 & 50 mins & 13.70 hours \\
\hline SRR019501 & $7,217,883$ & 52.0 & 52.5 & 26 mins & 11.94 hours \\
\hline SRR019597 & $5,943,586$ & 62.0 & 62.1 & 20 mins & 13.45 hours \\
\hline
\end{tabular}




\section{Availability and requirements}

- Project name: BS Seeker

- Project home page: http://pellegrini.mcdb.ucla.edu/ BS_Seeker/BS_Seeker.html

- Operating system(s): Linux, Mac OS

- Programming language: Python

- Other requirements: Python 2.5.2 or higher, Bowtie 0.10 .0 or higher

- License: Free for all users

- Any restrictions to use by non-academics: None, the software is readily available to any scientist wishing to use it for non-commercial purposes.

- The software (source code) and the examples are attached in Additional file 2 and Additional file 3, respectively. It can also be downloaded via the project home page.

\section{Additional material}

Additional file 1 Supplementary Information; supplementary materials to the BS Seeker project.

Additional file 2 BS Seeker software: a compressed file containing the source code for BS Seeker.

Additional file $\mathbf{3}$ Examples for running BS Seeker. This compressed file includes two example datasets (reads and the reference genome) for testing BS Seeker. A step by step instruction is included in Readme and can be also viewed from the project webpage at http://pellegrini.mcdb.ucla.edu/ BS Seeker/EXAMPLES.html.

\section{Authors' contributions}

PC wrote BS Seeker and drafted the manuscript. SC and MP participated in the design and helped to draft the manuscript. All authors read and approved the final manuscript.

\section{Acknowledgements}

The authors thank Krishna Chodavarapu for testing the program. PC is supported by Eli \& Edythe Broad Center of Regenerative Medicine \& Stem Cell Research at UCLA.

\section{Author Details}

Department of Molecular, Cell, and Developmental Biology, University of California, Los Angeles, CA, USA

Received: 30 December 2009 Accepted: 23 April 2010

Published: 23 April 2010

\section{References}

1. Frommer M, McDonald LE, Millar DS, Collis CM, Watt F, Grigg GW, Molloy $\mathrm{PL}$, Paul CL: A genomic sequencing protocol that yields a positive display of 5-methylcytosine residues in individual DNA strands. Proc Natl Acad Sci USA 1992, 89(5):1827-1831.

2. Cokus SJ, Feng S, Zhang X, Chen Z, Merriman B, Haudenschild CD, Pradhan S, Nelson SF, Pellegrini M, Jacobsen SE: Shotgun bisulphite sequencing of the Arabidopsis genome reveals DNA methylation patterning. Nature 2008, 452(7184):215-219.

3. Lister R, O'Malley RC, Tonti-Filippini J, Gregory BD, Berry CC, Millar AH, Ecker JR: Highly integrated single-base resolution maps of the epigenome in Arabidopsis. Cell 2008, 133(3):523-536.

4. Meissner A, Mikkelsen TS, Gu H, Wernig M, Hanna J, Sivachenko A, Zhang X, Bernstein BE, Nusbaum C, Jaffe DB, Gnirke A, Jaenisch R, Lander ES: Genome-scale DNA methylation maps of pluripotent and differentiated cells. Nature 2008, 454(7205):766-770.

5. Smith ZD, Gu H, Bock C, Gnirke A, Meissner A: High-throughput bisulfite sequencing in mammalian genomes. Methods 2009, 48(3):226-232.
6. Lister R, Pelizzola M, Dowen RH, Hawkins RD, Hon G, Tonti-Filippini J, Nery JR, Lee L, Ye Z, Ngo QM, Edsall L, Antosiewicz-Bourget J, Stewart R, Ruotti V, Millar AH, Thomson JA, Ren B, Ecker JR: Human DNA methylomes at base resolution show widespread epigenomic differences. Nature 2009, 462(7271):315-322.

7. Kent WJ: BLAT--the BLAST-like alignment tool. Genome Res 2002 12(4):656-664.

8. Li R, Li Y, Kristiansen K, Wang J: SOAP: short oligonucleotide alignment program. Bioinformatics 2008, 24(5):713-714.

9. Langmead B, Trapnell C, Pop M, Salzberg SL: Ultrafast and memoryefficient alignment of short DNA sequences to the human genome. Genome Biol 2009, 10(3):R25.

10. Xi Y, Li W: BSMAP: whole genome bisulfite sequence MAPping program. BMC Bioinformatics 2009, 10:232.

11. Smith AD, Chung W, Hodges E, Kendall J, Hannon G, Hicks J, Xuan Z, Zhang MQ: Updates to the RMAP short-read mapping software. Bioinformatics 2009

12. Li H, Ruan J, Durbin R: Mapping short DNA sequencing reads and calling variants using mapping quality scores. Genome Res 2008, 18(11):1851-1858.

13. Hodges E, Smith AD, Kendall J, Xuan Z, Ravi K, Rooks M, Zhang MQ, Ye K, Bhattacharjee A, Brizuela L, McCombie WR, Wigler M, Hannon GJ, Hicks JB: High definition profiling of mammalian DNA methylation by array capture and single molecule bisulfite sequencing. Genome Res 2009, 19(9):1593-1605.

14. Dohm JC, Lottaz C, Borodina T, Himmelbauer H: Substantial biases in ultra-short read data sets from high-throughput DNA sequencing. Nucleic Acids Res 2008, 36(16):e105.

doi: 10.1186/1471-2105-11-203

Cite this article as: Chen et al., BS Seeker: precise mapping for bisulfite sequencing BMC Bioinformatics 2010, 11:203

Submit your next manuscript to BioMed Centra and take full advantage of:

- Convenient online submission

- Thorough peer review

- No space constraints or color figure charges

- Immediate publication on acceptance

- Inclusion in PubMed, CAS, Scopus and Google Scholar

- Research which is freely available for redistribution 